\title{
Associação entre risco de transtornos mentais comuns e insegurança alimentar entre mães com filhos menores de um ano de idade
}

\author{
Association between minor mental disorders and household \\ feeding insecurity among mothers of children \\ less than one-year-old
}

Sarah Queiroga de Sousa (https://orcid.org/0000-0002-3882-0982) ${ }^{1}$

Ianna Karolina Véras Lôbo (https://orcid.org/0000-0001-5075-6859) ${ }^{2}$

Alice Teles de Carvalho (https://orcid.org/0000-0002-2915-8202) ${ }^{1}$

Rodrigo Pinheiro de Toledo Vianna (https://orcid.org/0000-0002-5358-1967) ${ }^{1}$
${ }^{1}$ Programa de PósGraduação em Ciências da

Nutrição, Departamento de Nutrição, Centro de Ciências da Saúde, Universidade Federal da Paraíba. Campus Universitário s/n, Castelo Branco III. 58059-900 João Pessoa PB Brasil. sarah.nutri@hotmail.com ${ }^{2}$ Programa de Pediatria e Ciências Aplicadas à Pediatria, Universidade Federal de São Paulo Disciplina de Nutrologia. São Paulo SP Brasil.

\begin{abstract}
Household food insecurity (HFI) is related to health and nutritional problems, however there are few extant studies that relate it to mental health. The scope of this article is to associate HFI with the risk of minor mental disorders $(M M D)$ in mothers with one-year-old children. A prospective cohort was conducted with 194 mothers and their babies from birth onwards. HFI was measured fourth months after birth using the Brazilian Household Food Insecurity scale and MMD risk amongst mothers was measured by the Self-Reporting Questionnaire (SRQ-20) when their baby was one year old. Socio-economic and health status were used for control. HFI was present in $59.3 \%$ of the families. The MMD risk ratio was 1.59 (CI 95\%: 1.10 - 2.31) in HFI families compared with secure families. This relationship remains significant in the multiple logistic model, $O R=2.20$ (CI 95\%: 1.16-4.20), after controlling by socio-economic and health variables. HFI is an independent risk factor to maternal risk of MMD. It should be important to include strategies to reduce HFI by promoting maternal mental health and improving child-mother quality of life.

Key words Food and nutritional security, Mental health, Maternal-child health
\end{abstract}

Resumo Insegurança alimentar e nutricional está relacionada a problemas nutricionais e de saúde, entretanto poucos estudos a relacionam com saúde mental. O objetivo deste artigo é Investigar associação da insegurança alimentar familiar e risco de transtornos mentais comuns (TMC) em mães com filhos de um ano de idade. Estudo de coorte prospectivo com 194 mães e seus filhos desde o nascimento. Insegurança alimentar foi medida aos quatro meses pós-parto, com a Escala Brasileira de Insegurança Alimentar e risco de diagnóstico positivo de TMC nas mães ao final do primeiro ano da criança, com o Self Response Questionnaire (SRQ-20). Medidas socioeconômicas e de saúde foram utilizadas como controle. Das famílias, 59,3\% apresentavam insegurança alimentar, tendo razão de risco para diagnóstico de TMC de 1,59 (IC 95\%: 1,10 - 2,31), comparado com famílias em segurança alimentar. Após ajuste, modelo logístico múltiplo estimou $O R=$ 2,20 (IC 95\%: 1,16 - 4,20) para esta relação. Observou-se associação da insegurança alimentar familiar aos quatro meses pós-parto e risco de diagnóstico de TMC entre mães ao final do primeiro ano dos filhos. O enfrentamento da insegurança alimentar deve fazer parte das estratégias promotoras da saúde materna e da qualidade de vida materno-infantil.

Palavras-chave Segurança alimentar e nutricional, Saúde mental, Saúde materno-infantil 


\section{Introdução}

A insegurança alimentar ocorre quando uma família não consegue ter acesso regular e permanente a alimentos de qualidade e em quantidade suficiente ou quando, para tê-lo, compromete o acesso a outras necessidades essenciais ${ }^{1}$. Engloba desde a preocupação e/ou angústia ante a incerteza de dispor regularmente de comida, até a vivência de fome por não ter o que comer durante todo o dia².

São conhecidos os fatores de risco para a ocorrência de insegurança alimentar entre famílias brasileiras, sendo a baixa renda um dos mais importantes ${ }^{3,4}$. Revisão sistemática recente descreveu relações entre a insegurança alimentar e os índices antropométricos em crianças e obesidade em adultos, especialmente mulheres, assim como redução da qualidade da dieta das famílias ${ }^{5}$. Estas importantes relações descrevem o contexto de ocorrência da insegurança alimentar, porém pouco se sabe das consequências deste agravo nas condições de saúde e de vida da população.

A análise dos dados de dois ciclos de uma pesquisa nacional realizada nos EUA (NHANES 2003-2008) mostrou que adultos com insegurança alimentar aumentam o risco de ocorrência de doenças cardiovasculares ${ }^{6}$. Também uma revisão sistemática encontrou evidências de que a insegurança alimentar seria um mediador importante entre o cuidado materno e o desenvolvimento infantil, podendo comprometer o desenvolvimento físico e psíquico das crianças. O principal fator relacionado com a diminuição do cuidado seria a ocorrência de transtornos mentais comuns associados às experiências de insegurança alimentar familiar?

Os Transtornos Mentais Comuns (TMC) que podem afetar todas as pessoas são a depressão, o transtorno bipolar, o transtorno do pânico e o transtorno de ansiedade generalizada, abrangendo sintomas como insônia, fadiga, esquecimento, irritabilidade, dificuldades de concentração, queixas somáticas e sentimento de inutilidade. Estas condições estão diretamente relacionadas ao declínio da qualidade de vida e da qualidade das relações sociais ${ }^{8}$.

No Brasil, até o presente momento, não existem estudos que tenham investigado a associação entre a insegurança alimentar e o risco de TMC em mulheres mães de crianças pequenas. Considerando que tanto a insegurança alimentar como o risco de TMC são problemas muito prevalentes: a primeira afeta em torno de $22,6 \%$ da população brasileira, sendo mais de $50 \%$ das pessoas com rendimento menor que meio salário mínimo per capita $^{4}$ e o segundo, de acordo com estudo realizado na cidade de São Paulo, pode atingir quase a metade da população ao longo das suas vidas9. Estes problemas isoladamente já causam alto risco atribuível na população $0^{10}$, podendo ser muito mais grave a associação entre eles, reduzindo a qualidade de vida da população.

Desta forma o objetivo deste trabalho foi, em uma coorte de binômio mães/filhos, investigar a associação entre a situação de insegurança alimentar familiar e o risco de transtornos mentais comuns nas mães e medir o risco relativo e atribuível desta relação, considerando que a falta de acesso ao alimento e as privações em decorrência da insegurança alimentar podem ser importantes estressores que aumentam o risco de TMC entre as mães.

\section{Métodos}

Trata-se de um estudo do tipo coorte prospectivo, no qual os binômios mãe-filho foram identificados no momento da internação para o parto em duas maternidades públicas de João Pessoa-PB, Brasil, a saber: Instituto Cândida Vargas, unidade de Referência Norte- Nordeste e Maternidade Frei Damião, referência Estadual.

Estas são as duas principais maternidades públicas da cidade, respondem a em torno de $60 \%$ do total de partos ocorridos anualmente em João Pessoa e em torno de $50 \%$ dos partos das gestantes residentes no município, considerando todos os estabelecimentos de saúde ${ }^{11}$.

O seguimento foi composto de três etapas: o cadastramento das mães, uma visita domiciliar em torno dos quatro meses após o nascimento do bebê, e uma entrevista telefônica em torno de um ano após o nascimento (Figura 1).

Foram elegíveis para este estudo todas as parturientes que deram entrada nestas maternidades para os procedimentos de parto durante o período de junho a agosto de 2013 e que atendiam aos seguintes critérios de inclusão: idade entre 18 e 35 anos e residir em João Pessoa. Foram critérios de exclusão mães com problemas graves de saúde como mães HIV positivas, vítimas de violência, com doenças raras, mães de gêmeos, de crianças prematuras, com malformações congênitas ou com doenças metabólicas graves, pelo motivo de que estes agravos poderiam ser causas independentes de TMC.

Considerando nível de significância de 95\% e poder do teste de $80 \%$, ocorrência de risco de 


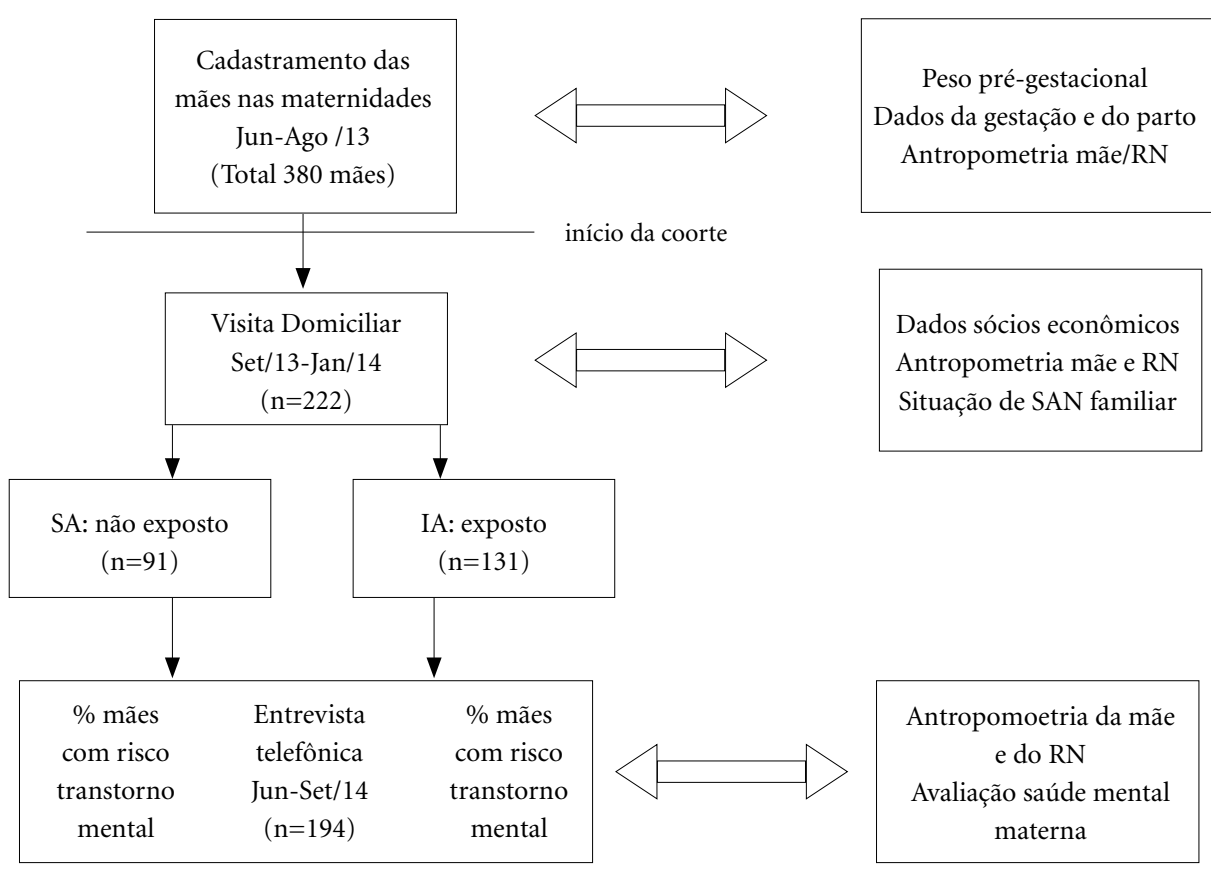

Figura 1. Fluxograma da coorte.

TMC em 20\% da população não exposta à insegurança alimentar, e razões de risco iguais ou superiores a 2, o número mínimo de participantes no estudo foi estimado em 182 binômios mãefilho ${ }^{12}$. A técnica de amostragem foi não probabilística, sendo incluídas na coorte todas as mães encontradas na visita domiciliar até completar o tamanho mínimo calculado acrescido de $20 \%$, o que resultou em uma amostra de 222 binômios mãe-filho. Permaneceram no estudo ao final do seguimento, 194 binômios mães-filhos que foram novamente entrevistados por meio de uma ligação telefônica. Os 28 casos de perda de seguimento foram devido à impossibilidade do contato telefônico, por aparelhos desligados (16), números não existentes (10) e não atendimento (2).

Toda coleta de dados foi realizada por uma equipe de campo treinada. Durante o cadastramento das mães, além do endereço e contato telefônico, foram observadas informações de prontuário sobre o número de consultas pré-natal, número de gestações, peso gestacional inicial e final. Foi registrado o tipo de parto e possíveis intercorrências da mãe ou do neonato.

Durante a visita domiciliar foi aplicado um questionário para informações socioeconômicas e a Escala Brasileira de Insegurança Alimentar, classificando as famílias em segurança alimentar ou insegurança alimentar nos níveis leve, moderado ou grave ${ }^{4}$. Foram aferidas as medidas antropométricas da mãe e do bebê $\hat{1}^{13,14}$. Para o lactente utilizou-se uma balança pediátrica eletrônica para aferir o peso e para a medição do comprimento utilizado foi um estadiômetro infantil confeccionado em madeira, medindo de zero a 100 centímetros. Para a avaliação antropométrica materna, foi feita aferição do peso e estatura utilizando uma balança plataforma digital portátil, com capacidade de $180 \mathrm{~kg}$ e uma fita métrica inelástica que foi fixada na parede, tomando-se o cuidado de escolher uma parede lisa, reta e sem rodapé visando menor influência na medida. Todas as medidas foram feitas em duplicata e nos casos de diferenças entre as medidas superiores a $0,5(\mathrm{~kg}$ ou $\mathrm{cm})$, uma terceira foi realizada e foram consideradas as duas mais próximas para o cálculo da média aritmética registrada. A classificação do estado nutricional das mães foi baseada no cálculo do Índice de Massa Corporal (IMC) ${ }^{15}$. Para as crianças foi considerado os agravos nutricionais, desnutrição ou excesso de peso, quando identificado desvios superiores a 2 escores $z$, ne- 
gativos ou positivos respectivamente dos indicadores peso para idade $(\mathrm{P} / \mathrm{I})$, comprimento para idade $(\mathrm{C} / \mathrm{I})$ e peso para o comprimento $(\mathrm{P} / \mathrm{C})$ comparado com os padrões de referência ${ }^{16}$.

A etapa seguinte foi realizada através de entrevista telefônica em que foram obtidas respostas referidas sobre o peso atual da mãe e das crianças, solicitando a informação do último registro de peso do cartão da criança. Finalmente, foi aplicado o instrumento de triagem Self Reporting Questionnaire - (SRQ-20) para rastreamento de transtornos não psicóticos entre as mães ${ }^{17}$. Ao final desta etapa de entrevista, os casos classificados como possível transtorno mental receberam orientação para encaminhamento às Unidades de Saúde de referência para a família.

Após a transcrição dos dados para meio digital, foi construído um arquivo para análise utilizando o programa estatístico SPSS for Windows ${ }^{\circledR}$, versão 19. Foi feita uma análise descritiva de todas as variáveis para corrigir as inconsistências observadas, retornando ao questionário original ou, quando necessário, buscando a informação junto à mãe entrevistada. Em casos que nenhuma destas ações foi possível, o dado foi considerado perdido. As mães incluídas na coorte foram comparadas com as demais do cadastramento para verificar se a amostragem foi tendenciosa utilizando teste qui-quadrado para as variáveis categóricas e teste de comparação de médias t-student para as variáveis contínuas, adotando-se nível de significância de 5\%.

A descrição da amostra foi feita com a frequência absoluta e relativa das variáveis categóricas e calculado as médias e os respectivos intervalos de confiança 95\% das variáveis numéricas contínuas. A variável dependente do estudo foi o resultado positivo de risco de TMC e a variável de exposição foi a situação de insegurança alimentar. A relação entre estas variáveis foi testada com a aplicação do teste qui-quadrado, considerando nível de significância de 95\%.

Para observar o efeito independente da insegurança alimentar no resultado de risco de TMC foi feita uma análise de regressão logística múltipla, na qual foram incluídas no modelo as variáveis de controle que apresentaram possível associação, com valor de $\mathrm{p}<0,20$ no teste quiquadrado, com a variável dependente do estudo.

A técnica utilizada para a construção do modelo logístico foi de inclusão progressiva das variáveis de acordo com a estatística Wald, considerando a probabilidade menor ou igual a 5\% para a variável ser incluída no modelo e $10 \%$ para ser excluída.
Foi calculada a fração atribuível na população da insegurança alimentar para o resultado positivo de TMC, baseado na diferença entre a prevalência total de risco de TMC em todo grupo estudado e a prevalência no grupo em segurança alimentar (não exposto). Em seguida calculou-se, em porcentagem, quanto esta diferença representa em relação à prevalência total de $\mathrm{TMC}^{10}$.

O projeto foi aprovado ao Comitê de Ética do Centro de Ciências da Saúde - UFPB. Todos os procedimentos foram adotados de acordo com a Resolução CNS no 466/12 que regulamenta pesquisas envolvendo seres humanos. Todas as mães receberam e assinaram TCLE no momento do cadastramento na maternidade.

\section{Resultados}

Completaram a coorte 194 binômios mãe-filho. Este grupo, quando comparado com as mães cadastradas na maternidade e que não compuseram a coorte, mostrou-se semelhante com relação às características registradas na maternidade a saber: mãe primípara ( $\mathrm{p}=0,503)$, ocorrência prévia de aborto ou perda fetal $(\mathrm{p}=0,264)$, tipo de parto $(\mathrm{p}=0,418)$, média de idade da mãe ( $\mathrm{p}$ $=0,905)$, ganho de peso durante a gestação $(\mathrm{p}$ $=0,340)$, número de consultas pré-natal ( $\mathrm{p}=$ $0,171)$, sexo do recém nascido $(\mathrm{p}=0,939)$, peso ao nascer $(\mathrm{p}=0,056)$, comprimento ao nascer $(\mathrm{p}=0,108)$ e perímetro cefálico $(\mathrm{p}=0,058)$. Ocorreram 28 perdas de seguimento $(12,6 \%)$ que quando foram comparadas com as mães que completaram o seguimento se mostraram semelhantes em todas características, com exceção de primiparidade, pois houveram mais perdas entre as mães com mais de um filho $(\mathrm{p}=0,018)$. Não houve diferença entre as perdas de seguimento com relação à avaliação de insegurança alimentar $(\mathrm{p}=0,493)$.

A idade média das mães na maternidade foi de 25,6 anos (IC 95\%: 25,0 - 26,3 anos). Do total, $43,8 \%$ eram primíparas e $63,2 \%$ dos partos foram cesarianos. Com relação à ancestralidade, $87,1 \%$ se autorreferiram pardas e, entre aquelas com relato de gestação anterior, 22,7\% referiram já haver sofrido aborto ou algum caso de morte fetal. Com relação às características socioeconômicas das mães, 95,3\% vivem em casas de alvenaria, o acesso a água é quase universal, sendo disponível diariamente para 75,8\% dos casos, quase a totalidade das moradias $(94,7 \%)$ relatam ter condições satisfatórias de esgotamento sanitário (fossa ou rede pública). As características socioe- 
conômicas e de saúde das mães e das crianças são mostradas na Tabela 1 .

A avaliação da situação de segurança alimentar e dos diferentes níveis de insegurança alimentar, realizada na visita domiciliar, classificou $40,7 \%$ das famílias em segurança alimentar, $45,9 \%$ em insegurança alimentar leve, 6,2\% em moderada e 7,2\% em grave. Em torno de um ano após o nascimento da criança, a frequência de resultados positivos para risco de TMC foi de $42,8 \%$ entre todas as mães do estudo. Estratificando as mães segundo a classificação prévia da situação de insegurança alimentar familiar observa-se que as prevalências de resultado positivo para TMC aumenta na medida em que as famílias passam de segurança para insegurança alimentar leve, moderada e grave (Figura 2).

Calculando-se a razão de risco bruta de resultado positivo de TMC em função da situação de insegurança alimentar observada aos quatro meses pós-parto, observa-se que ela foi significativa quando as famílias se encontravam com níveis moderado, $\mathrm{RR}=2,10(1,26-3,52)$ e grave, $\mathrm{RR}=$ 2,03 (1,22 - 3,37), comparado com famílias em segurança alimentar. Relacionando as famílias em segurança com as em insegurança alimentar, independente da sua gravidade, a razão de risco bruta de obter resultado positivo para TMC foi $1,59(1,10-2,31)$. Este último resultado apresentou poder do teste de $74,3 \%$, com aproximação da distribuição normal, sem correção para a continuidade da distribuição amostral ${ }^{17}$.

A possível associação das demais variáveis do estudo com o resultado positivo de TMC, adotando-se p-valor $<20 \%$, segundo teste qui-quadrado, indicou que: mãe responsável pelo domicílio, menor renda familiar (primeiro tercil), menor escolaridade materna, moradia não ser de alvenaria, domicílio com esgoto inadequado, mãe maior que 25 anos, mãe multípara, ter história de aborto ou morte fetal, filho com febre ou dor de ouvido ou excesso de peso no período da visita domiciliar aumentam a prevalência de TMC. Desta forma, todas estas variáveis foram incluídas no modelo de regressão logística múltiplo.

O modelo final teve a inclusão progressiva de três variáveis que obtiveram p-valor menor que 0,05: insegurança alimentar, história prévia de aborto ou morte fetal e excesso de peso do filho no momento da visita domiciliar. A Tabela 2 mostra os valores de Odds Ratio e os respectivos intervalos de confiança 95\% para as variáveis do modelo final.

Assim, mães que sofreram algum aborto ou perda fetal ao longo de sua história reprodutiva
Tabela 1. Características sociais, econômicas e de saúde dos binômios mãe - filho acompanhados na coorte. João Pessoa, 2014.

\begin{tabular}{|c|c|c|}
\hline Característica & $\mathbf{N}$ & $\%$ \\
\hline \multicolumn{3}{|l|}{ Responsável pelo domicílio } \\
\hline Marido & 115 & 59,6 \\
\hline Mulher & 15 & 7,8 \\
\hline Avós ou outros & 63 & 32,6 \\
\hline \multicolumn{3}{|l|}{ Ocupação Materna } \\
\hline Do lar & 118 & 60,8 \\
\hline Algum trabalho remunerado & 76 & 39,2 \\
\hline \multicolumn{3}{|l|}{ Escolaridade materna } \\
\hline Fundamental & 55 & 28,5 \\
\hline Médio & 120 & 62,2 \\
\hline Superior & 18 & 9,3 \\
\hline \multicolumn{3}{|l|}{ Renda } \\
\hline $1^{\circ}$. tercil renda pc (até $\left.0,26 \mathrm{SM}^{\star}\right)$ & 63 & 33,0 \\
\hline $2^{\circ}$. tercil renda $\mathrm{pc}\left(0,26\right.$ até $\left.0,48 \mathrm{SM}^{\star}\right)$ & 63 & 33,0 \\
\hline $3^{\circ}$.tercil renda $\mathrm{pc}\left(0,48\right.$ até $\left.2,30 \mathrm{SM}^{\star}\right)$ & 65 & 34,0 \\
\hline Estado nutricional materno (4 meses) & 5 & 2,7 \\
\hline$<18,5$ - baixo peso & 73 & 39,9 \\
\hline $18,5-24,9$ - adequado & 65 & 35,5 \\
\hline $25,0-29,9$ - sobrepeso & 40 & 21,9 \\
\hline \multicolumn{3}{|l|}{$>=30$ - obesidade } \\
\hline \multicolumn{3}{|l|}{ Estado nutricional materno ( 1 ano) } \\
\hline$<18,5$ - baixo peso & 7 & 3,7 \\
\hline $18,5-24,9$ - adequado & 77 & 41,0 \\
\hline $25,0-29,9$ - sobrepeso & 68 & 36,2 \\
\hline$>=30-$ obesidade & 36 & 19,1 \\
\hline Estado nutricional do bebê (4 meses)- P/I & 164 & 89,1 \\
\hline$<+2$ z-escore & 20 & 10,9 \\
\hline \multicolumn{3}{|l|}{$>=+2$ z-escore } \\
\hline \multicolumn{3}{|l|}{ Estado nutricional do bebê ( 1 ano)- P/I } \\
\hline$<+2$ z-escore & 142 & 77,6 \\
\hline$>=+2 \mathrm{z}$-escore & 41 & 22,4 \\
\hline
\end{tabular}

Tabela 2. Odds Ratio e intervalo de confiança 95\% das variáveis relacionadas com o aumento da prevalência de resultados positivos para risco de TMC nas mães da coorte. João Pessoa, 2014.

\begin{tabular}{lrc}
\hline \multicolumn{1}{c}{ Variável } & OR & IC 95\% \\
\hline Sem história prévia de aborto & 1 & \\
História de aborto ou perda fetal & 2,39 & $1,15-4,98$ \\
Filho peso eutrófico (4 meses) & 1 & \\
Filho com excesso de peso (4 meses) & 2,85 & $1,04-7,82$ \\
Segurança alimentar (4 meses) & 1 & \\
Insegurança alimentar (4 meses) & 2,20 & $1,16-4,20$ \\
\hline
\end{tabular}

tiveram 2,39 vezes mais chances de terem resultado de risco para TMC; a criança ter excesso de peso nos primeiros meses de vida também foi 


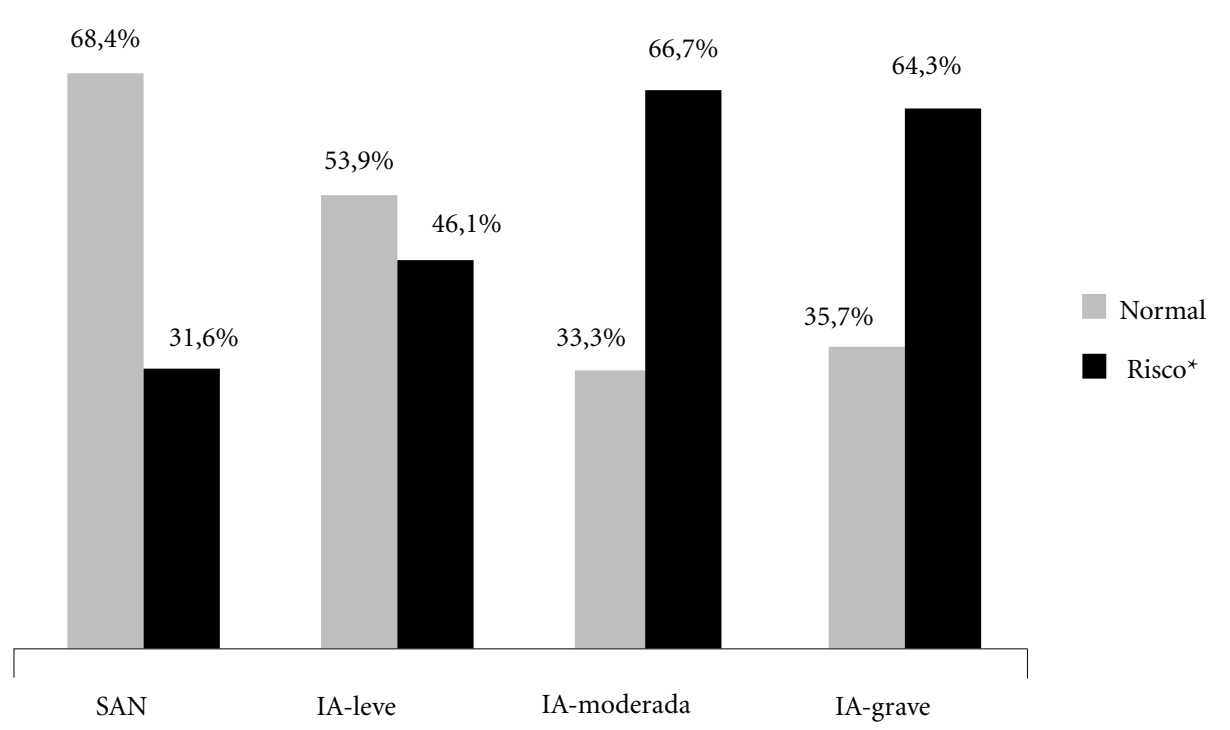

Figura 2. Prevalência de diagnóstico de risco de TMC em função da situação de segurança e dos diferentes níveis de insegurança alimentar. João Pessoa, 2014.

${ }^{*}$ p-valor $=0,0019$ teste Qui-Quadrado de Tendência Linear, considerando a situação de segurança e os três níveis de insegurança alimentar (Mantel-Haenszel).

fator de risco, aumentando 2,85 vezes a chance para o resultado positivo de risco de TMC em torno de 12 meses após o parto e finalmente, a experiência vivenciada de insegurança alimentar aumentou a chance de resultado de risco de TMC em 2,20 vezes, quando comparado com aquelas mães que pertenciam a famílias em segurança alimentar no mesmo período do tempo.

A fração atribuível da insegurança alimentar para a ocorrência dos resultados positivos de risco de TMC é a diferença entre a prevalência de TMC na população total $(42,8 \%)$ e a prevalência no grupo em segurança alimentar $(31,6 \%)$, e o seu valor corresponde a 11,2\%. Em porcentagem, este valor corresponde a uma redução percentual de $26,1 \%$ da prevalência da classificação de risco para TMC em mulheres com filhos menores de um ano de idade se a situação de insegurança alimentar de suas famílias fosse resolvida.

\section{Discussão}

O presente estudo encontrou uma associação entre a situação de insegurança alimentar vivenciada pela família nos primeiros meses após a ges- tação associado com maior risco de ocorrência de transtornos mentais comuns nas mães ao final do primeiro ano de vida do seu filho, entretanto deve-se considerar que uma limitação deste trabalho foi o fato destas duas medidas haverem sido realizadas em momentos diferentes, a EBIA, referente aos três meses anteriores foi aplicada quatro meses depois do parto e o SRQ-20 aplicado 12 meses após o parto, de modo que estes resultados não refletem o mesmo período de cada mãe acompanhada.

Mas os resultados deste trabalho mostraram forte associação entre estes dois eventos, insegurança alimentar e risco de TMC, bem como um efeito dose-resposta observado com aumento progressivo do risco de TMC à medida que aumentam os graus de insegurança alimentar domiciliar comparativamente às mães que residem em domicílios com segurança alimentar, sendo evidências de confirmação da relação entre estes dois agravos de saúde.

Esta relação, também, é bastante plausível se considerarmos que na sociedade atual os mecanismos, tanto de sobrevivência como para a inserção social, em qualquer estrato socioeconômico, são influenciados pela alimentação, assim é de 
se esperar a insegurança alimentar cause transtornos além do campo físico, biológico ou nutricional. Estudo longitudinal realizado no Reino Unido com mais de mil famílias com crianças pequenas, para verificar o papel da insegurança alimentar no desenvolvimento cognitivos das crianças, mostrou que a sensibilidade materna para identificar as necessidades das crianças é diminuída nas famílias em insegurança alimentar, afetando então o desenvolvimento intelectual e afetivo das crianças ${ }^{18}$. Estudo prospectivo realizado nos Estados Unidos com mulheres beneficiárias de um programa social observou que insegurança alimentar grave, denominada insuficiência de alimentos, aumenta as taxas de depressão entre mulheres de baixo nível socioeconômico, depois de controlar o efeito de outros fatores conhecidos que aumentam o risco de transtornos mentais como a pobreza e a exclusão social ${ }^{19}$. Estudo longitudinal inglês, realizado com 1.116 famílias, observou que nas de baixa renda, a presença de transtornos mentais maternos está relacionada com maior insegurança alimentar, mostrando a relação bidirecional destes dois fenômenos ${ }^{20}$. Finalmente, estudo específico para verificar a associação de insegurança alimentar com sintomas depressivos foi realizado com gestantes latinas vivendo em comunidades de baixa renda no Estado de Connecticut, Estados Unidos, e confirmou que aquelas que vivem em famílias em insegurança alimentar têm quase três vezes mais chances de sofrer sintomas depressivos comparado com as gestantes de famílias em segurança alimentar, controlando por outros fatores de risco ${ }^{21}$. Todos estes exemplos confirmam a associação apresentada pelo presente estudo, sugerindo a possibilidade de ser uma relação de causalidade.

A principal limitação para o estabelecimento de relação causal está no fato das mães da coorte não haverem sido investigadas com relação ao seu risco de diagnóstico positivo de risco de TMC aos quatro meses, junto com a medida da exposição à insegurança alimentar, bem como a escala de avaliação da insegurança alimentar não ter sido aplicada no final do seguimento. A primeira limitação é mais grave, pois os estudos de coorte pressupõe a ausência de doença no início do seguimento, entretanto optou-se por não aplicar o referido instrumento em função da proximidade do parto e as preocupações e os cuidados do puerpério poderiam enviesar os resultados positivos de ansiedade ou depressão medidos pelo SRQ-2022.

O segundo problema, a modificação do status de insegurança alimentar também é possí- vel que tenha ocorrido ao longo dos oito meses que transcorreram entre as medidas, entretanto é possível também que os efeitos da insegurança alimentar vivenciada não desapareçam mesmo nos casos da sua diminuição. Para conhecer mais sobre estes mecanismos novos estudos necessitam ser realizados. Também, caso a exposição tenha sido menor ao longo do período, ainda assim os resultados foram significativos. Este fato, acompanhado com o efeito dose resposta observado, sugere que o risco de resultado positivo para TMC devido à situação de insegurança alimentar pode vir a ser maior do que o descrito neste trabalho, porém não menor.

É relevante que até a presente data nenhum estudo foi realizado observando a relação entre a segurança alimentar e a saúde mental materna na população brasileira, embora trabalhos já tenham confirmado a relação entre saúde mental materna e cuidado infantil, como, por exemplo, o estudo transversal com mães e seus respectivos filhos de um ano de idade, atendidas em Unidades Básicas de Saúde, que identificou que TMC são fator de risco para o desenvolvimento infantil ${ }^{23}$.

$O$ presente estudo encontrou quase a metade das mães com escores positivos no teste SRQ-20 para TCM. Esta frequência é em torno de 30\% nas mães vivendo em segurança alimentar, chegando a um pouco mais de $60 \%$ no grupo daquelas em insegurança alimentar grave. Alguns estudos de base populacional foram realizados no Brasil investigando a prevalência de risco de TMC utilizando o mesmo instrumento de avaliação. No município de Pelotas, RS, no ano de 2005, observou-se $32,8 \%$ de resultados positivos de risco de TMC entre mulheres, sendo que, entre aquelas de menor renda, a prevalência subiu para $43,7 \%{ }^{24}$. Em Recife, no ano de 2006, 1.120 gestantes foram investigadas para verificar a relação entre a ocorrência de risco de TMC e violência por parceiro íntimo durante a gravidez. O grupo de mulheres que relatou não haver sofrido violência apresentou 33,8\% de risco de TMC, enquanto aquelas que relataram sofrer violência física ou sexual, com ou sem violência psicológica, apresentaram $71,0 \%$ de risco de $\mathrm{TMC}^{25}$. Estes resultados de investigações com mulheres, na região Sul do Brasil ou na região Nordeste, confirmam as altas prevalências observadas de risco de TMC na população brasileira e que são muito semelhantes àquelas observadas no presente estudo. Situações graves como a privação de renda ou a própria violência aumentam as prevalências de TMC nas mulheres. Por analogia, a insegurança alimentar vivenciada é também um evento 
importante do ponto de vista da sua gravidade e das suas consequências que reduzem a qualidade de vida da população.

Considerando o fator de risco estudado, a insegurança alimentar familiar, a prevalência observada nas famílias das mães deste estudo, no momento da entrevista domiciliar, foi de 59,3\%. Este valor foi superior à última estimativa nacional obtida na Pesquisa Nacional de Amostragem Domiciliar-PNAD, realizada em 2013, na qual observou-se a prevalência de $36,5 \%$ de insegurança alimentar no estado da Paraíba ${ }^{4}$. Considerando que o grupo estudado não representa a população do município ou do estado, a comparação com dados de inquéritos de base populacional é limitada. Entretanto, por se tratar de uma população de nível socioeconômico baixo, SUS dependente, é de se esperar prevalências de insegurança alimentar superiores à média estadual. De fato, observando a situação de insegurança alimentar por classe de rendimento, famílias com renda per capita média entre 0,5 e 1,0 salários mínimos na Paraíba, segundo levantamento da PNAD, apresentaram $12,7 \%$ de insegurança moderada ou grave $^{4}$, percentual semelhante aos $13,4 \%$ observado no nosso estudo, em que a renda média das famílias foi de 0,52 salários mínimos.

Outras variáveis associadas ao risco de TMC que permaneceram no modelo foram a resposta positiva para a mãe haver sofrido algum aborto ou perda fetal ao longo de sua história reprodutiva, e o recém-nascido estar com excesso de peso no momento da visita domiciliar, avaliado através das medidas diretas realizadas no domić́lio. O primeiro evento já foi descrito em outros trabalhos com possibilidade confirmada de manifestação de sintomas e distúrbios psicológicos meses ou anos após a vivência de uma a perda ${ }^{26,27}$. A segunda variável incorporada no modelo pode estar relacionada a características da alimentação da criança que não foi investigada, como a prática reduzida de aleitamento materno (que por sua vez atua no vínculo mãe filho), no reconhecimento materno e social do cuidado, bem como na possível substituição de alimentos adequados por alimentos menos nutritivos, porém com maior densidade energética. Todas estas possibilidades refletiriam outras faces da insegurança alimentar, que é o foco principal deste trabalho.

A variável renda não foi incluída no modelo, apesar de ser uma variável de controle importante que está associada à ocorrência de inúmeras doenças, bem como insegurança alimentar e os TMC. Isto deve ter ocorrido por se tratar de uma amostra com pouca variação de renda, entretan- to é importante considerar a necessidade de se investigar este efeito em estudos futuros.

Este estudo de seguimento utilizou diferentes métodos de coleta de dados ao longo do tempo de seguimento da coorte, o que pode trazer algum viés aos resultados, especialmente ao que se refere à memória das mães para relatar o peso pré-gestacional, quando o mesmo não estava registrado no cartão da gestante, às respostas de morbidade referida da criança ou respostas obtidas por telefone, no momento da última entrevista. Nesta, tratou-se de utilizar um formulário bastante objetivo e curto, com poucas questões além do SRQ-20 e ainda solicitando que a mãe fizesse uso do cartão da criança para minimizar erros. O Brasil tem uma experiência positiva de inquéritos telefônicos ${ }^{28-30} \mathrm{e}$ este fato reforça nossa confiança de que estas limitações estão longe de inviabilizar os resultados encontrados neste trabalho.

Finalmente, os resultados ajustados deste trabalho mostram uma chance aumentada $(\mathrm{OR}=$ $2,20)$ de risco de TMC em mães pertencentes a famílias que vivenciaram insegurança alimentar. Isto é especialmente relevante considerando que esta situação afeta $22,6 \%$ das famílias brasileiras $^{13}$. Porém, considerando somente o universo das mães SUS dependentes, que neste estudo apresentaram prevalência muito superior de insegurança alimentar, pode-se afirmar que o impacto da redução da insegurança alimentar para a diminuição de ocorrência de risco de TMC é muito grande ${ }^{10}$.

\section{Conclusão}

O estudo descreve uma consequência importante da insegurança alimentar que é sua relação com a saúde mental materna podendo causar transtornos mentais comuns como as ansiedades e depressões. Além da importância deste fato para a saúde pública, ajuda a entender novos elementos do conceito de insegurança alimentar, relacionado a aspectos de cidadania e garantias sociais, além daqueles biológicos e nutricionais.

Considerando as altas prevalências de insegurança alimentar e seu impacto no risco de TMC, a prevenção e o cuidado aos transtornos mentais maternos deve considerar o enfrentamento da insegurança alimentar como forma de minimizar este problema. A redução da insegurança alimentar familiar como dos transtornos mentais das mães durante o primeiro ano de vida de seus filhos deverá ter impactos positivos na qualidade 
de vida destas famílias como também possibilitar melhores cuidados à criança pequena, aumentando seu potencial de desenvolvimento na vida que se inicia.

\section{Colaboradores}

SQ Sousa - Realizou o trabalho de campo, participou das análises e interpretação dos resultados e fez a principal redação do manuscrito. IKV Lôbo - Participou do planejamento do estudo, realizou trabalho de campo, participou das análises iniciais dos dados e realizou a revisão do manuscrito. AT Carvalho - Participou do planejamento do estudo e colaborou na revisão final do manuscrito. RPT Vianna - Realizou a concepção do trabalho, participou do planejamento do estudo, das análises estatísticas dos dados, redação do manuscrito e revisão da versão final do manuscrito.

\section{Referências}

1. Brasil. Presidência da República. Lei no 11.346 , de 15 de setembro de 2006. Cria o Sistema Nacional de Segurança Alimentar e Nutricional - SISAN com vistas em assegurar o direito humano à alimentação adequada $\mathrm{e}$ dá outras providências. Diário Oficial da União 2006; 16 set.

2. Pérez-Escamilla R, Segall-Corrêa AM, Kurdian Maranha L, Sampaio MFA, Marin-Leon L, Panigassi G. An adapted version of the U.S. Department of Agriculture Food Insecurity module is a valid tool for assessing household food insecurity in Campinas, Brazil. J Nutr 2004; 134(8):1923-1928.

3. Cabral CS, Lopes AG, Lopes JM, Vianna RPT. Segurança alimentar, renda e Programa Bolsa Família: estudo de coorte em municípios do interior da Paraíba, Brasil, 2005-2011. Cad Saude Publica 2014; 30(2):393-402.

4. Instituto Brasileiro de Geografia e Estatística (IBGE). Pesquisa Nacional por Amostra de Domicílios - Segurança Alimentar 2013. Rio de Janeiro: IBGE; 2014.

5. Morais DC, Dutra LV, Franceschini SCC, Priore SE. Insegurança alimentar e indicadores antropométricos, dietéticos e sociais em estudos brasileiros: uma revisão sistemática. Cien Saude Colet 2014; 19(5):1475-1488.

6. Ford ES. Food Security and Cardiovascular Disease Risk Among Adults in the United States: Findings From the National Health and Nutrition Examination Survey, 2003-2008. Prev Chronic Dis 2013; 10(202):15451551.

7. Perez-Escamilla R, Vianna RPT. Food Insecurity and the Behavioral and Intellectual Development of Children: A review of the evidence. J Appl Res Child: Informing Policy for Children at Risk 2012; 3(1):9.

8. Brunoni AR. Transtornos mentais comuns na prática clínica. Rev Med 2008; 87(4):251-263.

9. Andrade L, Walters EE, Gentil V, Laurenti R. Prevalence of ICD-10 mental disorders in a catchment area in the city of Sao Paulo, Brazil. Soc Psychiatry Psychiatr Epidemiol 2002; 37(7):316-325.

10. Szklo M. Epidemiologia translacional: algumas considerações. Epidemiol Serv Saude 2015; 24(1):161-172.

11. Paraíba. Secretaria de Estado da Saúde [homepage da internet]. Nascimentos - Frequência segundo estabelecimentos de Saúde. SES-PB/GEVS/GORR/SIM e SINASC [acessado 2015 Jun 8]. Disponível em: http://www.saude.pb.gov.br

12. Dean AG, Sullivan KM, Soe MM. OpenEpi: Open Source Epidemiologic Statistics for Public Health, Versão 3.03. [homepage da internet]. [acessado 2015 Maio 4]. Disponível em: www.OpenEpi.com. 
13. World Health Organization (WHO). Physical Status: the use and interpretation of anthropometry. Genebra: WHO; 1995. [Technical Report Series 854].

14. Brasil. Ministério da Saúde (MS). Orientações para coleta e análise de dados antropométricos em serviços de saúde: Norma técnica do Sistema de Vigilância Alimentar e Nutricional - SISVAN. Brasília: MS; 2011.

15. World Health Organization (WHO). Physical status: The use and interpretation of anthropometry. Geneva: WHO; 1995. [Report of a WHO expert committee].

16. World Health Organization (WHO). WHO Child Growth Standards. Length/height for age, weight for age, wheight for length, wheight for height and body mass index for age: methods and development. Genebra: WHO; 2006.

17. World Health Organization (WHO). A user's guide to the Self Reporting Questionnaire (SRQ). Geneva: WHO; 1994.

18. Belsky DW, Moffitt TE, Arseneault L, Melchior M, Caspi A. Context and sequelae of food insecurity in children's development. Am J Epidemiol 2010; 172(7):809818.

19. Heflin CM, Siefert K, Williams DR. Food insufficiency and women's mental health: findings from a 3-year panel of welfare recipients. Soc Sci Med 2005; 61(9):1971-1982.

20. Melchior M, Caspi A, Howard LM, Ambler AP, Bolton H, Mountain N, Moffitt TE. Mental health context of food insecurity: a representative cohort of families with young children. Pediatrics 2009; 124(4):564-572.

21. Hromi-Fiedler A, Bermúdez-Millán A, Segura-Pérez S, Pérez-Escamilla R. Household food insecurity is associated with depressive symptoms among low-income pregnant Latinas. Matern Child Nutr 2011; 7(4):421430.

22. Noble RE. Depression in women. Metabolism 2005; 54(5 Supl. 1):49-52.

23. Ribeiro DG, Perosa GB, Padovani FHP. Fatores de risco para o desenvolvimento de crianças atendidas em Unidades de Saúde da Família, ao final do primeiro ano de vida: aspectos sociodemográficas e de saúde mental materna. Cien Saude Colet 2014; 19(1):215-226.
24. Anselmi L, Barros FC, Minten GC, Gigante DP, Horta BL, Victora CG. Prevalência e determinantes precoces dos transtornos mentais comuns na coorte de nascimentos de 1982, Pelotas, RS. Rev Saude Publica 2008; 42(Supl. 2):26-33.

25. Ludermir AB, Valongueiro S, Araújo TVB. Common mental disorders and intimate partner violence in pregnancy. Rev Saude Publica 2014; 48(1):29-35.

26. Couto ER, Couto E, Vian B, Gregório Z, Nomura ML, Zaccaria R, Passini Júnior R. Quality of life, depression and anxiety among pregnant women with previous adverse pregnancy outcomes. Sao Paulo Med J 2009; 127(4):185-189.

27. Lok IH, Neugebauer R. Psychological morbidity following miscarriage. Best Pract Res Clin Obstet Gynaecol 2007; 21(2):229-247.

28. Brasil. Ministério da Saúde (MS). VIGITEL. Brasil 2012: Vigilância de fatores de risco e proteção para doenças crônicas por inquérito telefônico. Brasília: MS; 2013.

29. Malta DC, Andrade SC, Claro RM, Bernal RTI, Monteiro, CA. Evolução anual da prevalência de excesso de peso e obesidade em adultos nas capitais dos 26 estados brasileiros e no Distrito Federal entre 2006 e 2012. Rev Bras Epidemiol 2014; 17(Supl. 1):267-76.

30. Francisco PMSB, Barros MBA, Segri NJ, Alves MCGP. Comparação de estimativas de inquéritos de base populacional. Rev Saude Publica 2013; 47(1):60-68.

Artigo apresentado em 05/04/2017

Aprovado em 28/08/2017

Versão final apresentada em 30/08/2017 